\section{Verus Ring as a New Device to Perform a Safe and Efficient Capsulorhexis in Patients with Corneal Opacities Undergoing Cataract Surgery}

Received: February 07, 2017; Accepted: February 20, 2017; Published: February 27, 2017

\section{Background and Introduction}

One of the most difficult and critical step in cataract surgery is making a perfectly circular and properly sized capsulorhexis, allowing the capsular bag to completely envelop uniformly the IOL optic, reducing the incidence of posterior capsular opacification and providing a more predictable effective lens position [1]. And to prevent anterior capusle extension which complicates cataract surgery and might prevent completion of cataract surgery especially in cases that visibility is not optimal like in corneal scars.

Some techniques or devices has been developed in order to make this step easier as the Verus ring (Verus capsulorhexis device, Mile High Ophthalmics), a silicone ring-shaped first suggested as an easy-to-use device option for guiding surgeons under training to perform anterior circular curvilinear capsulorhexis, allowing an efficient opening in the anterior capsule, sizing and safe centering a continuous curvilinear capsulorhexis with minimal cost compared to a femtosecond laser [2] which can do the same job but with more cost. Many studies support the theory of the importance of perfect sizing and centering the capsulorhexis on effect lens position.

Capsulorhexis is a delicate step during phacoemulsification requiring experience of the surgeon and can be even more challenging in corneal opacities. We are proposing a new use for the Verus ring to perform capsulorhexis in patients with corneal opacity.

Verus ring has been pioneered by Dr. Malik Y. Kahook who is the Glaucoma service chief, University of Colorado, Aurora, Colorado.

\section{Methods}

Eyes with mild to moderate corneal scars undergoing cataract surgery $(n=20)$ were subjected to implantation of the Verus ring during capsulorhexis by an expert single surgeon. Outcome measures included complications during the capsulorhexis procedure and time taken to complete the capsulorhexis and surgeon feedback. Historical and surgeon controls $(n=20)$ for capsulorhexis in patients with clear cornea were used as a comparative group.

\section{Enrique Suarez ${ }^{1}$ and Trad Alkadi ${ }^{1,2}$ \\ 1 King Khalid Eye Specialist Hospital, Saudi Arabia \\ 2 Imam Muhammad Ibn Saud Islamic University, Saudi Arabia}

Corresponding author: Enrique Suarez

esuarez@kkesh.med.sa

King Khalid Eye Specialist Hospital, Saudi Arabia.

Citation: Suarez E, Alkadi T. Verus Ring as a New Device to Perform a Safe and Efficient Capsulorhexis in Patients with Corneal Opacities Undergoing Cataract Surgery. J Intensive \& Crit Care 2017, 3:1.

In our study group, the patients were 11 male and 9 female. Age range was from 44-70 years old with mean age of 59 years old. Thirteen of our patients (65\%) were diabetic, while 7 patients ( $35 \%$ ) were medically free. We tried to select our control group from the historical to be comparative in demographic data to our study group. The data from both groups were comparable with no statistically significant difference between both groups (Table 1).

\section{Surgical Technique}

The anterior chamber needs to be completely filled with viscoelastic prior to Verus ring can be injected in the anterior chamber through a $2.2 \mathrm{~mm}$ surgical incision (main phacoemulsification incision), then the ring is positioned over the anterior capsule pressuring it gently with a lens manipulator instrument. The anterior chamber should be refilled with dispersive OVD to help adding more pressure over the Verus ring to indent over the anterior capsule (Figure 1). The capsulorhexis

\begin{tabular}{|c|c|c|}
\hline & Study population & Control \\
\hline Mean Age & 59 & 61 \\
\hline Age Range & $44-70$ & $46-72$ \\
\hline Gender & $\mathrm{M}=11, \mathrm{~F}=9$ & $\mathrm{M}=12, \mathrm{~F}=8$ \\
\hline Diabetic & $13(65 \%)$ & $12(60 \%)$ \\
\hline Medically free & $7(35 \%)$ & $8(40 \%)$ \\
\hline
\end{tabular}

Table 1 Control group from the historical to be comparative in demographic data. 
flap is starting by tearing the central part of the lens anterior capsule, then lifting it up toward the internal edge of the Verus device with a capsulorhexis forceps. The capsular flap is walked along the inner edge of the Verus ring while being lifted slightly over the device so that the cut in the capsule is occurring in a manner similar to "ripping a piece of paper against an overlying ruler" [2].

\section{Results}

There were no complications related to the insertion and removal of the device. Anterior capsular extensions were not observed in any of the cases with corneal scarring (Zero\%). The capsulorhexis was well centered in all the eyes with $5.5 \mathrm{~mm}$. The average time for capsulorhexis using the Verus ring was 25.59 s. Average historical and surgeon control time to perform capsulorhexis was $28.21 \mathrm{~s}$. This difference was $2.62 \mathrm{~s}$.

Surgeon feedback indicated that the insertion of the device did not require steep learning curve other than careful following the instructions as noted in the instructional video and was safe, easier, less stressful and better centration and curvilineal even with the presence of corneal scar (Figure 2).

\section{Conclusion}

The Verus ring appears to be a useful device in performing safe and efficient capsulorhexis in patients with corneal scars undergoing cataract surgery. It is also a useful device for physicians under training and residents as it adds safety and centration and perfect sizing. We suggest using Verus ring in cases of mild to moderate corneal scars.
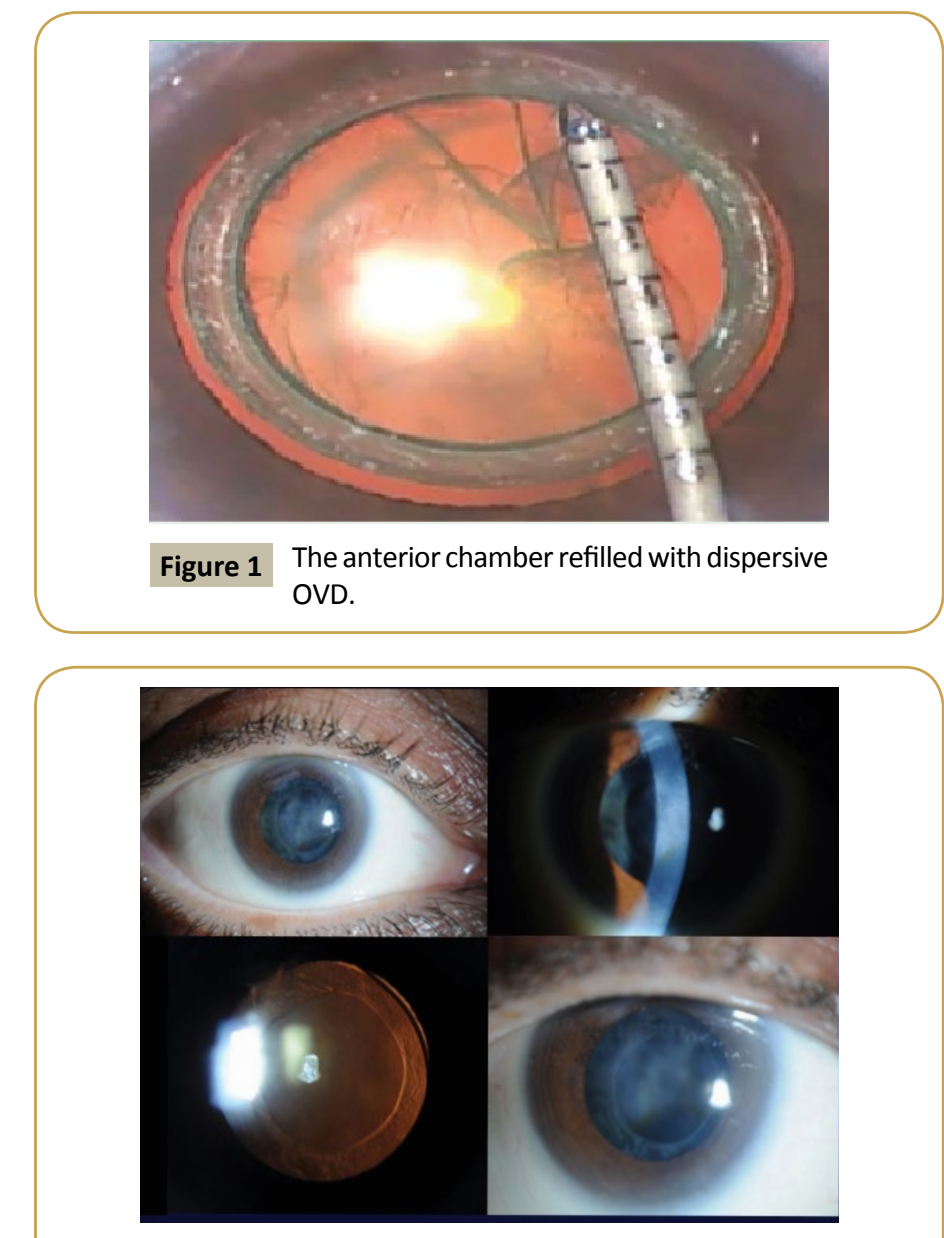

Figure 2 Postoperative appearance of curvilinear continuous capsulorhexis with scars in the cornea. 


\section{References}

1. Mohammadpour AH, Shamsara J, Nazemi S, Ghadirzadeh S, Shahsavand S, et al. (2012) Evaluation of RANKL/OPG serum concentration ratio as a new biomarker for coronary artery calcification: A pilot study. Thrombosis 2012: 306263.

2. Powers MA, Kahook MY (2014) New device for creating a continuous curvilinear capsulorhexis. J Cataract Refract Surg 40: 822-830. 